\title{
"Hand-Over-Hand Grasping Technique": A Fast and Safe Procedure for Specimen Extraction in Laparoscopic Sleeve Gastrectomy
}

\author{
Georges Bou Nassif $^{1} \cdot$ Elsa Scetbun $^{1} \cdot$ Cyntia Lecurieux-Lafayette $^{1} \cdot$ Andrea Lazzati $^{1}$
}

Published online: 27 February 2017

(C) Springer Science+Business Media New York 2017

\begin{abstract}
Background Laparoscopic sleeve gastrectomy (LSG) has become one of the most popular bariatric surgeries worldwide, given good result concerning weight loss and long-term morbidity.

While surgical technique is well standardized, specimen extraction is still a matter of controversy between surgeons.

In this video, we present a simple, fast, safe, and reproducible technique of specimen extraction after laparoscopic sleeve gastrectomy.

Methods After the sleeve gastrectomy is completed with hemostasis, the specimen is introduced in a retrieval bag. Stomach specimen orientation is very important, such as the largest specimen part is removed firstly. The $2 \mathrm{~cm}$ of the held fundus is introduced under direct vision in the $12-\mathrm{mm}$ trocar. Traction is applied to simultaneously remove the trocar, the cord of the bag, and
\end{abstract}

the grasper. The stomach is held by a Kocher once it is outside the peritoneal cavity. The bag is opened and everted to protect the wound. The pneumoperitoneum is deflated to relax the abdominal wall. The specimen is removed from the abdominal cavity by traction handover-hand grasping with gauze.

Results We have applied this technique in 200 consecutive patients with an average body mass index of 42.5. At the first postoperative visit (1 month), none of the patients experienced wound infection. Clinical incisional hernia was detected in one patient $(0.5 \%)$.

Conclusions This hand-over-hand grasping with gauze technique is simple, safe, reproducible, and time saving.

Keywords Sleeve gastrectomy · Specimen extraction · Laparoscopy $\cdot$ Trocar port hernia $\cdot$ Trocar port infection

Electronic supplementary material The online version of this article (doi:10.1007/s11695-017-2572-2) contains supplementary material, which is available to authorized users.

Georges Bou Nassif

george.bn@hotmail.com

Elsa Scetbun

elsascetbun@gmail.com

Cyntia Lecurieux-Lafayette

cynthia.lecurieuxlafayette@gmail.com

Andrea Lazzati

andrea.lazzati@chicreteil.fr

1 Department of Digestive Surgery, Centre Hospitalier Intercommunal de Créteil, 40 Avenue de Verdun, 94000 Créteil, France 